\title{
Gastrointestinal Complications in Two Neonates
}

Rakhi Gupta Basuray, MD - Devon Swick, DO - Ansley Splinter, MD, MAcM

Two unrelated male neonates were born at term in the same hospital on the same day. Both mothers had prenatal care established in the first trimester, and both pregnancies were uncomplicated. Maternal serologic testing and the routine anatomy ultrasonographic scans were normal for both. The delivery of both neonates was uncomplicated with normal Apgar scores at $1 \mathrm{~min}$ and $5 \mathrm{~min}$. Vitamin $\mathrm{K}$ and erythromycin were administered to both infants. The initial physical examination of both neonates was within normal limits.

\section{Clinical Course of Neonate 1}

At 9 hours of life, after attempts at breastfeeding, 2 episodes of bilious, nonbloody emesis occurred. The infant appeared well, alert, and in no distress. His abdomen was soft, nontender, and nondistended, with active bowel sounds. An abdominal radiography scan revealed gaseous distention of bowel or colonic loops in the left abdomen, which may represent a developing obstruction (Figure 1). The neonate was monitored closely, and within 2 hours, another episode of

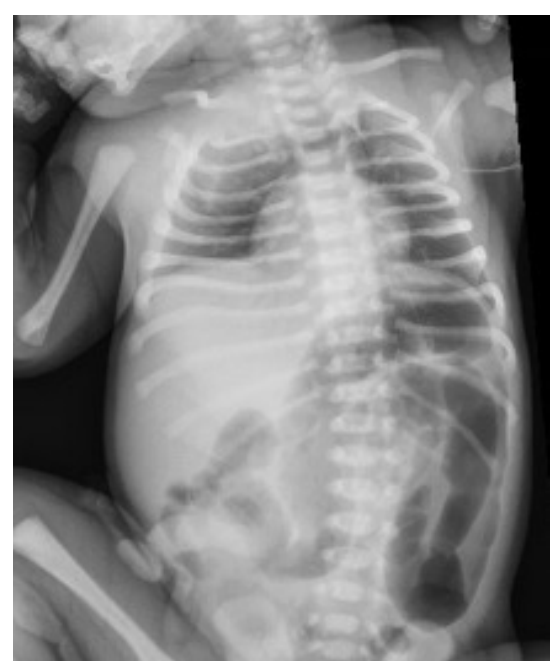

Figure 1. An abdominal radiography scan of neonate 1 demonstrated gaseous distension of the descending colon, sigmoid colon, and rectum, with a sigmoid colon more distended than the rectum.

bilious, nonbloody emesis occurred, with an increase in his abdominal circumference by $1.5 \mathrm{~cm}$.

\section{Clinical Course of Neonate 2}

At 12 hours of life, after breast milk

\section{AFFILIATIONS:}

'Department of Pediatrics, The Ohio State University College of Medicine, Columbus, Ohio ${ }^{2}$ Nationwide Children's Hospital, Columbus, Ohio

\section{CITATION:}

Gupta Basuray RG, Swick D, Splinter A. Gastrointestinal complications in two neonates. Consultant. Published online May 26, 2021. doi:10.25270/con.2021.05.00012

Received January 20, 2021. Accepted March 29, 2021.

\section{DISCLOSURES:}

The authors report no relevant financial relationships.

\section{CORRESPONDENCE:}

Rakhi Gupta Basuray, MD, Division of Hospital Medicine, Nationwide Children's Hospital and the Ohio State University, 700 Children's Dr, Columbus, OH 43205 (Rakhi.GuptaBasuray@nationwidechildrens.org)

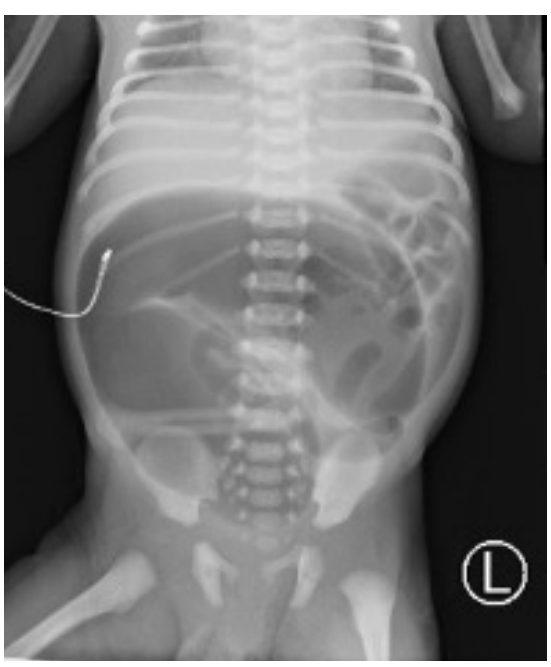

Figure 2. An abdominal radiography scan of neonate 2 demonstrated a C-shaped, markedly distended, bowel loop in the central abdomen with a narrowing of the distal small bowel.

and formula supplement feeding, small episodes of nonbilious, nonbloody emesis occurred. Similar to neonate 1 , the boy initially appeared well, with a soft, nontender, nondistended abdomen and normoactive bowel sounds. But by 16 hours of life, projectile emesis developed with a distended, tender abdomen. An abdominal radiography scan showed dilated loops of bowel with a narrowing of the distal small bowel (Figure 2).

Both neonates were deemed to receive nothing by mouth and had orogastric catheters placed to decompress their stomachs, as well as an intravenous line. Both neonates were transferred to a higher level of care for further management.

\section{Discussion}

Pediatric colorectal conditions encompass a broad spectrum of differential 
diagnoses. They range from intussusception in infants to inflammatory bowel disease in adolescents. This report visually compares and contrasts the clinical and radiographic presentation of 2 distinct obstructive processes identifiable in the immediate neonatal period.

Hirschsprung disease (HD), famously known for the absence of ganglionic cells in the gastrointestinal tract, is a condition affecting approximately 1 out of every 5000 neonates. As a result of the neural crest cells failing to adequately develop and migrate proximally, segments of bowel extending from the rectum to the small intestine may be affected. 'The involved segment is most often limited to the rectosigmoid region in $80 \%$ of patients, with male patients affected 3 to 4 times more often than female patients. Most cases occur sporadically, but nearly $30 \%$ are associated with chromosomal or congenital anomalies such as Down syndrome or congenital central hypoventilation syndrome. ${ }^{2}$

As a result of impaired peristalsis, half of cases are identified in the first year of life. Interestingly, while $95 \%$ of neonates with normal bowel function will pass meconium in the first 24 hours of life, $90 \%$ of patients with HD will as well. ${ }^{1}$ However, $40 \%$ of HD cases will be diagnosed in patients by 6 months of age, $60 \%$ by 2 years of age, and $80 \%$ by 7 years of age. Detection can be subtle but progress to include growth failure and refractory constipation dependent on rectal enemas and suppositories. ${ }^{2}$ On occasion, diagnosis is made after a critical presentation of enterocolitis, involving abdominal tenderness, leukocytosis, and hematochezia. ${ }^{1}$

Colonic atresia (CA) is much rarer than $H D$, with an incidence of approximating 1 in every 50,000. Specific findings on antenatal ultrasonography scans are lacking, making prenatal diagnosis difficult.

Presentation is classically within the first 48 hours of life and includes failure to pass meconium, new onset abdominal distension, or bilious emesis. ${ }^{3}$

Diagnosis of both HD and CA require radiography scans and contrast

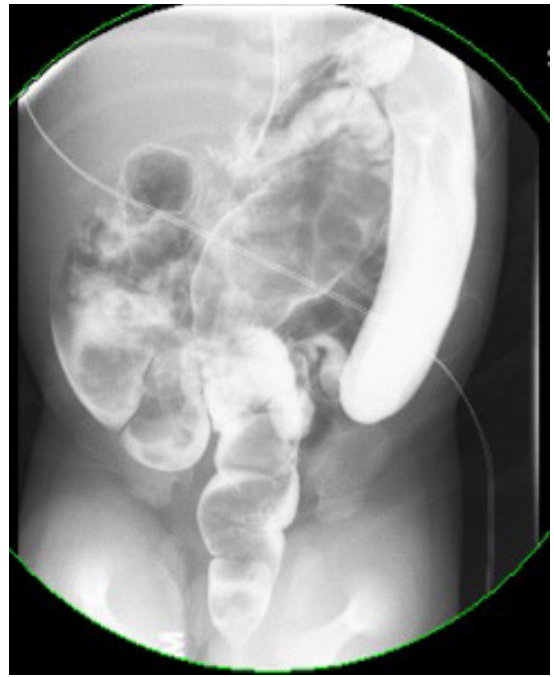

Figure 3. Contrast enema demonstrated a larger sigmoid colon than rectum, as seen in patients with Hirschsprung disease.

enema. For neonates, the primary goal of abdominal imaging is to identify the presence of an obstructive pattern. It is not otherwise radiographically possible to distinguish small bowel from large bowel in this population. The presence of double or triple bubbles would be concerning for a high obstructive process and would require follow-up with an upper gastrointestinal series. On the other hand, large loops of gas-filled bowel in the shape of "tubes" would suggest a low obstruction and necessitate further imaging with a water-soluble contrast enema. This latter modality can then distinguish microcolon as a ribbon-like, continuous, and uniformly small segment of colon from a "transition zone" in which there is change in colonic caliber with a narrow, diseased segment distal to a normal, distended region of the bowel. ${ }^{4}$

In cases of HD, a colorectal region full of stool can be visualized on the radiograph, while the retrograde enema allows for a more thorough evaluation and approximation of the transition zone. 'Since the rectum normally serves as a reservoir larger than the sigmoid colon, HD can be suggested when this ratio is reversed on contrast enema (Figure 3 ). ${ }^{4} \mathrm{~A}$ diagnostic low-risk suction biopsy of the mucosa

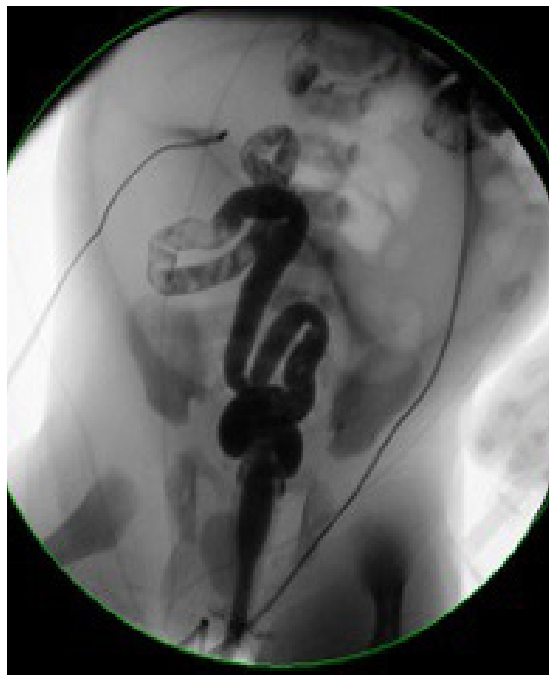

Figure 4. Contrast enema demonstrated microcolon with an inability to reflux contrast into the known dilated loops, as seen in patients with colonic atresia.

and submucosa can be performed in infants at the bedside, but it requires full-thickness samples via open rectal biopsy in older patients. Adjunctively, anorectal manometry may demonstrate an absent rectoanal inhibitory reflex, whereby the distension of the rectum is not associated with relaxation of the internal sphincter.

In cases of $\mathrm{CA}$, radiography scans are likely to show a dilated loop of bowel closed at the ileocecal valve on one end and the proximal site of atresia at the other end. Flow on contrast enema is expected to arrest distal to the atretic region, without refluxing into the dilated loop, with the additional visualization of microcolon (Figure 4). Laparoscopic exploration can confirm the diagnosis. ${ }^{5}$

Management of both conditions requires nasogastric decompression, intravenous fluid hydration and, ultimately, surgical intervention. A single-stage, minimally invasive, transanal approach is most common for HD. The aganglionic region is resected followed by anastomosis of the proximal ganglionic segment with the distal rectum. Continence can be preserved when anastomosis occurs above the dentate line, without encroachment into the anal canal and sphincter com- 
plex. In cases of CA, excision with anastomosis may be adequate for right-sided findings, while a staged approach with an initial ostomy may be needed for transverse or left-sided colonic atresia. ${ }^{3}$

$\mathrm{CA}$ and HD carry a co-occurrence rate of about $2 \%$ to $8.4 \% .{ }^{6}$ If the latter is undiagnosed, surgical outcomes of colonic atresia can be significantly impacted. As such, biopsies to rule out HD are advised before completing anastomotic repair of colonic atresia. Overall prognosis of colonic atresia hinges on the morbidity and mortality of associated conditions. ${ }^{6}$

\section{Treatment and management}

Postoperative follow-up of patients with HD allows ongoing monitoring for the development of enterocolitis, as well as for the possibility of retained aganglionic segments of bowel. Enterocolitis can be treated with bowel rest, intravenous antibiotics, and rectal irrigations. ${ }^{1}$ Ongoing obstructive symptoms can be treated with injections of botulinum toxin into the anal sphincter with complication rates less than $1 \%$ after a pull-through procedure, as first described in 1997. ${ }^{7}$ The prognosis for normal bowel functioning without constipation, overflow incontinence, or dependence on pharmacologic therapies remains unknown. Although, parents/guardians of patients with HD report similar quality of life scores as parents/guardians of healthy children. ${ }^{8}$

\section{Patient outcomes}

The outcomes of both neonates have been favorable thus far. HD was diagnosed in neonate 1. He underwent a pullthrough procedure with subsequent botulinum toxin injection and was prescribed as-needed rectal irrigations. CA was diagnosed in neonate 2. He underwent an ileostomy formation with mucus fistula, and a repair procedure with ileostomy takedown at 6 months of life is planned.

\section{References}

1. Gourlay DM. Colorectal considerations in pediatric patients. Surg Clin N Am. 2013;93(1):251-272. https://doi. org/10.1016/j.suc.2012.09.017

2. Ambartsumyan L, Smith C, Kapur RP. Diagnosis of Hirschsprung disease. Pediatr Dev Pathol. 2020;23(1):8-22. https://doi. org/10.1177/1093526619892351

3. Adams SD, Stanton MP. Malrotation and intestinal atresias. Early Hum Dev. 2014;90(12):921-925. https://doi. org/10.1016/j.earlhumdev.2014.09.017

4. Tsitsiou $Y$, Calle-Toro JS, Zouvani A, Andronikou S. Diagnostic decision-making tool for imaging term neonatal bowel obstruction. Clin Radiol. 2021;76(3):163-171. https://doi. org/10.1016/j.crad.2020.09.016

5. Zhou J-L, Fang Y-L, Tian S, Zhu X-C, Ge W-P. Radiological feature of colonic atresia. Arch Dis Child Fetal Neonatal Ed. 2018;103(3):F263. https://doi.org/10.1136/ archdischild-2017-314482

6. El-Asmar KM, Abdel-Latif M, El-Kassaby AA, Soliman MH, El-Behery MM. Colonic atresia: association with other anomalies. J Neonat Surg. 2016;5(4):47-52. https://doi. org/10.21699/jns.v5i4.422

7. Halleran DR, Lu PL, Ahmad H, et al. Anal sphincter botulinum toxin injection in children with functional anorectal and colonic disorders: a large institutional study and review of the literature focusing on complications. J Pediatr Surg. 2019;54(11):23052310. https://doi.org/10.1016/j.jpedsurg.2019.03.020

8. Townley OG, Lindley RM, Cohen MC Murthi GV. Functional outcome, quality of life, and 'failures' following pull-through surgery for Hirschsprung's disease: a review of practice at a single-center. $J$ Pediatr Surg. 2020;55(2):273-277. https:// doi.org/10.1016/j.jpedsurg.2019.10.042 